\title{
Intervenants en protection de la jeunesse : exploration des motifs de carrière initiaux, des sources de motivation actuelles et des répercussions perçues de ces sources de motivation actuelles
}

\section{Youth protection workers: Exploration of initial career reasons, current sources of motivation, and perceived impacts of these sources}

\author{
Stéphanie Marchand, Nathalie Houlfort, Amélie Couvrette, Stéphane Guay et \\ Steve Geoffrion
}

Volume 47, numéro 2, 2018

URI : https://id.erudit.org/iderudit/1054059ar

DOI : https://doi.org/10.7202/1054059ar

Aller au sommaire du numéro

Éditeur(s)

Revue de Psychoéducation

ISSN

1713-1782 (imprimé)

2371-6053 (numérique)

Découvrir la revue

Citer cet article

Marchand, S., Houlfort, N., Couvrette, A., Guay, S. \& Geoffrion, S. (2018). Intervenants en protection de la jeunesse : exploration des motifs de carrière initiaux, des sources de motivation actuelles et des répercussions perçues de ces sources de motivation actuelles. Revue de psychoéducation, 47(2), 231-253. https://doi.org/10.7202/1054059ar
Résumé de l'article

objectif. L'objectif de cette étude qualitative est de décrire la motivation au travail des intervenants en protection de la jeunesse (PJ) en explorant leurs motifs initiaux de carrière (pourquoi avoir choisi de travailler en PJ) ainsi que leurs sources de motivation actuelles (les irritants et les avantages perçus dans leur environnement de travail). Les répercussions négatives et positives perçues de ces sources de motivation actuelles ont également émergé. Méthode. Trente intervenants en PJ ont participé à une entrevue téléphonique semi-structurée. Une analyse thématique séquenciée a été réalisée en vue d'identifier les thèmes émergents. Résultats. Les intervenants choisissent de travailler en PJ pour des motifs opportunistes (par exemple., circonstances de vie) et des motifs relationnels (par exemple., aider les jeunes). Les participants se sont aussi prononcés sur les irritants et les avantages perçus dans leur environnement de travail. À cet effet, au niveau organisationnel, les intervenants en protection de l'enfance disent, entre autres éléments, ne pas aimer la présence de violence dans leur milieu de travail. Toutefois, au plan relationnel, les participants rapportent aimer beaucoup le fait d'être témoin du cheminement des clients. Lors de l'analyse, les répercussions négatives et positives des sources de motivation actuelles ont émergé. Par exemple, compte tenu des éléments décrits comme irritants dans l'environnement, certains intervenants disent souhaiter quitter l'organisation. À l'inverse, les intervenants disent que les éléments avantageux dans leur environnement de travail les amènent à apprécier davantage leur mandat de travail. Discussion. Il semble judicieux d'élaborer un environnement de travail en PJ supportant plus la relation d'aide auprès de la clientèle. Un tel environnement permettrait de mieux satisfaire les besoins psychologiques des intervenants et soutenir leur motivation au travail, puis de réduire les difficultés de santé psychologique et de roulement de personnel.
Tous droits réservés ( La Corporation de la Revue Canadienne de Psycho-Éducation, 2018
Ce document est protégé par la loi sur le droit d'auteur. L’utilisation des services d’Érudit (y compris la reproduction) est assujettie à sa politique d'utilisation que vous pouvez consulter en ligne.

https://apropos.erudit.org/fr/usagers/politique-dutilisation/ 


\section{Intervenants en protection de la jeunesse : exploration des motifs de carrière initiaux, des sources de motivation actuelles et des répercussions perçues de ces sources de motivation actuelles}

\section{Youth protection workers: Exploration of initial career reasons, current sources of motivation, and perceived impacts of these sources}

\section{S. Marchand ${ }^{1}$ \\ N. Houlfort ${ }^{1}$ \\ A. Couvrette ${ }^{2}$ \\ S. Guay ${ }^{3}$ \\ S. Geoffrion ${ }^{3}$}

1 Département de psychologie, Université du Québec à Montréal

2 Département de psychoéducation et de psychologie, Université du Québec en Outaouais

3 École de criminologie, Université de Montréal

4 École de psychoéducation, Université de Montréal

\section{Correspondance :}

Stéphanie Marchand, Université du Québec à Montréal, Département de Psychologie,

C.P. 8888, succ. Centre-ville, Montréal (Québec) H3C 3P8

marchand.stephanie.2@courrier. uqam.ca

Tél. : 514 508-5779

\section{Résumé}

Objectif. L'objectif de cette étude qualitative est de décrire la motivation au travail des intervenants en protection de la jeunesse $(P J)$ en explorant leurs motifs initiaux de carrière (pourquoi avoir choisi de travailler en $P J)$ ainsi que leurs sources de motivation actuelles (les irritants et les avantages perçus dans leur environnement de travail). Les répercussions négatives et positives perçues de ces sources de motivation actuelles ont également émergé. Méthode. Trente intervenants en PJ ont participé à une entrevue téléphonique semi-structurée. Une analyse thématique séquenciée a été réalisée en vue d'identifier les thèmesémergents. Résultats. Lesintervenants choisissent de travailler en PJ pour des motifs opportunistes (par exemple., circonstances de vie) et des motifs relationnels (par exemple., aider les jeunes). Les participants se sont aussi prononcés sur les irritants et les avantages perçus dans leur environnement de travail. À cet effet, au niveau organisationnel, les intervenants en protection de l'enfance disent, entre autres éléments, ne pas aimer la présence de violence dans leur milieu de travail. Toutefois, au plan relationnel, les participants rapportent aimer beaucoup le fait d'être témoin du cheminement des clients. Lors de l'analyse, les répercussions négatives et positives des sources de motivation actuelles ont émergé. Par exemple, compte tenu des éléments décrits comme irritants dans l'environnement, certains intervenants disent souhaiter quitter l'organisation. À l'inverse, les intervenants disent que les éléments avantageux dans leur environnement de travail les amènent à apprécier davantage leur mandat

1. Cette étude a été soutenue par l'octroi de bourses d'études décernées au dernier auteur par les Fonds de la Recherche du Québec - Société et culture (FRQSC), I'Institut de recherche Robert-Sauvé en santé et sécurité au travail (IRSST), le Centre international de criminologie comparée (CICC), la fondation de l'Institut universitaire en santé mentale de Montréal ainsi que par la subvention d'équipe des Instituts de recherche sur la santé du Canada (TVG251591) décernée à l'équipe de recherche VISAGE (violence au travail selon le sexe et le genre). 


\title{
232
}

de travail. Discussion. II semble judicieux d'élaborer un environnement de travail en PJ supportant plus la relation d'aide auprès de la clientèle. Un tel environnement permettrait de mieux satisfaire les besoins psychologiques des intervenants et soutenir leur motivation au travail, puis de réduire les difficultés de santé psychologique et de roulement de personnel.

\section{Mots-clés : motivation au travail, intervenants en protection de la jeunesse, roulement du personnel, problèmes de santé psychologique}

\begin{abstract}
Objective. The objective of this qualitative study is to describe how the work environment affects the motivation of youth protection workers (YPWs) by exploring their initial motives (why I chose to be a YPW), their actual motives (what are irritants and advantages in my work environment) and the impact of these motives. Methods. A sample of 30 YPWs was invited to participate in a semi-structured telephone interview. A sequenced thematic analysis was carried out in order to identify the emerging themes. Results. YPWs choose to work in their field for opportunistic (e.g., life circumstances) and relational (e.g., helping young people) motives. Organizational factors, including the presence of violence in the workplace, are perceived as demotivating. Relational factors, such as witnessing client progress, are seen as highly sources of motivating. The desire to leave the organization is one of the negative consequences of irritants present in the work environment of the YPW, while appreciating one's mandate is an example of the positive impact of advantages in work environment. Discussion. It appears wise to develop a work environment for YPWs that lends greater support to the act of intervening with youth. Such an environment would better satisfy the psychological needs of the YPWs, support their motivation at work and reduce psychological health issues and staff turnover.
\end{abstract}

Keywords: motivation at work, youth protection workers, staff turnover, psychological health problems

\section{Introduction}

Les travailleurs en protection de la jeunesse (PJ) sont à haut risque d'éprouver des problèmes de santé psychologique tels qu'un trouble de stress post-traumatique (TSPT; Horwitz, 2006; Rhee, Ko et Han, 2013) ou un épuisement professionnel (Anderson, 2000; Bennett, Plint et Clifford, 2005). Des études (Meadors et Lamson, 2008; Regehr, Hemsworth, Leslie, Howe et Chau, 2004) montrent que cet état de fait est partiellement attribuable à l'environnement de travail de la PJ. À titre d'exemple, dans un tel environnement, les intervenants sont exposés de façon récurrente à du matériel traumatique (Ferguson, 2005) et à de la violence (Geoffrion et Ouellet, 2013; Littlechild, 2005a, 2005b; Littlechild, Hunt, Goddard, Cooper, Raynes, et Wild, 2016; Macdonald et Sirotich, 2005).

Outre les conséquences sur les travailleurs mêmes, les problèmes de santé psychologique au travail affectent le fonctionnement des organisations de par leurs effets sur la performance, l'absentéisme, les accidents de travail et le roulement de personnel (Harnois et Gabriel, 2000). À cet égard, plusieurs études (Drake et Yadama, 1996; Ellet, Ellis, Westbrook et Dews, 2007; Mor Barak, Levin, Nissly et Lane, 2006) ont observé un haut taux de roulement de personnel en PJ. Une étude québécoise réalisée auprès de 586 éducateurs en PJ révèle qu'un intervenant sur trois songe à quitter son emploi (Geoffrion et Ouellet, 2013). II va sans dire qu'en 
plus des coûts financiers importants (Graeff et Hill, 2000), le taux de roulement a des effets considérables sur la prestation de services auprès de la clientèle, car il implique une rotation des dossiers parmi différents intervenants (Flower, McDonald et Sumski, 2005; Tremblay et Joly, 2009).

Ces études soulèvent l'importance de mieux comprendre le fondement des problèmes de santé psychologique au travail et de roulement de personnel en PJ. À notre connaissance, la plupart des études identifient les déterminants organisationnels tels que la violence ou le manque de support, des problèmes de santé psychologique au travail (par exemple., Bell, Kulkarni et Dalton, 2001; Geoffrion et Ouellet, 2013) et de roulement de personnel (par exemple., Mor Barack, Nissly et Levin, 2001). Pourtant, des recherches (par exemple., Diefendorff, Houlfort, Vallerand et Krantz, sous presse; Laframboise, Laurent et Houlfort, 2016; Otis et Pelletier, 2005) suggèrent que l'étude de la motivation au travail permettrait de mieux comprendre ces phénomènes. Par exemple, l'étude d'Otis et Pelletier (2005), effectuée auprès de 140 policiers francophones, suggère que la motivation au travail de type autodéterminé soit associée au bien-être des travailleurs et au désir de demeurer en emploi. Dans un contexte où les problèmes de santé psychologique et de roulement de personnel sont préoccupants, la motivation au travail représente un sujet d'intérêt pour les organisations en protection de l'enfance puisque maintes études suggèrent qu'elle influence le bien-être psychologique (par exemple., Fernet, Austin et Vallerand, 2012; Nie, Chua, Yeung, Ryan et Chan, 2014; Trépanier, Fernet et Austin, 2013) et l'intention de demeurer en emploi (par exemple., Gillet, Gagné, Sauvagère et Fouquereau, 2013; Otis et Pelletier, 2005).

Ainsi, le peu d'études sur la motivation au travail en protection de l'enfance, la gravité des problèmes de santé psychologique et de roulement de personnel soulèvent la nécessité de mieux comprendre la motivation au travail des intervenants. S'appuyant sur les résultats d'une étude réalisée auprès de 30 intervenants en PJ, cet article vise le développement de connaissances sur la motivation au travail dans le domaine de la protection de l'enfance en explorant les motifs ayant incité les individus à entreprendre une carrière en PJ et les sources de motivation actuelles.

\section{La protection de la jeunesse comme environnement de travail}

En PJ, les intervenants font régulièrement face à une clientèle peu volontaire et non collaborative, car ils sont amenés à intervenir à la suite d'un signalement (Ellet et al., 2007; Horejsl, Garthewait et Rolando, 1994). Ils interviennent auprès de l'enfant et de sa famille dans le but de les aider et de s'assurer du respect de l'application des diverses lois en matière juvénile. Ils interviennent dans des cas variés (par exemple., cas d'abandon, de maltraitance physique ou psychologique, d'abus sexuels), complexes (par exemple., troubles de santé mentale et d'abus de substances chez le parent) et nombreux (U.S. Government Accountability Office, 2003). Ces travailleurs sont exposés à d'importantes responsabilités et ils sont imputables de l'ensemble des décisions qu'ils prennent (Geoffrion, Morselli et Guay, 2016; Osofsky, Putnam et Lederman, 2008). À cet effet, maints intervenants en PJ vivent de l'anxiété en lien avec leur emploi (Gibbs, 2001). 
Outre les importantes préoccupations vécues, les intervenants en PJ peuvent être victimes ou témoins d'actes de violence commis par les jeunes (ou les parents) à leur égard. La revue de littérature de Lanctôt et Guay (2014) propose que la violence en milieu de travail engendre plusieurs conséquences néfastes, soit des problèmes de santé mentale (par exemple., TSPT) et une diminution de la productivité, de l'engagement et de la satisfaction au travail des employés. Les résultats de l'étude de Geoffrion et Ouellet (2013) confirment, en partie, ces résultats en indiquant que la violence physique et le climat de travail occasionné par la violence augmentent l'absentéisme et le désir des intervenants en PJ de quitter leur emploi. Mor Barak et al. (2001) suggèrent que certains autres éléments dans l'environnement de travail de la PJ, comme le stress découlant d'une surcharge de travail, contribuent à alimenter l'intention des travailleurs de quitter leur organisation.

Selon le modèle « Job Demands-Resources » (Bakker, Demerouti, De Boer et Schaufeli, 2003; Bakker, Demerouti, Taris, Schaufeli et Schreurs, 2003; Demerouti, Bakker, Nachreiner et Schaufeli, 2000, 2001), ces éléments font en sorte que le travail d'intervenant en PJ est un emploi constitué de demandes élevées. Jumelés à un environnement qui offre peu de ressources pour faire face à ces demandes, ces facteurs peuvent contribuer à une piètre santé psychologique au travail, à une réduction de l'engagement et à des intentions de quitter l'organisation. Bien que ce modèle soit intéressant pour expliquer pourquoi certains emplois et certains milieux peuvent miner ou contribuer au mieux-être des individus, il ne permet pas de cerner les mécanismes psychologiques sous-jacents permettant d'expliquer de tels effets. L'étude de Fernet et al. (2012), réalisée auprès de 586 directeurs d'école, a permis de mieux comprendre ces mécanismes. Basés sur la théorie de l'autodétermination (Ryan et Deci, 2017), Fernet et collègues (2012) ont examiné le rôle de la motivation comme variable médiatrice entre les ressources disponibles d'un emploi et le bienêtre de directeurs d'école. Leurs résultats montrent que les ressources d'un emploi prédisent positivement la motivation autodéterminée au travail, une forme positive de motivation, et négativement la motivation contrôlée, une forme de motivation moins optimale. Ces résultats suggèrent que les ressources (et les demandes) associées à un emploi influencent le bien-être et l'engagement grâce aux effets sur la motivation des travailleurs, mettant ainsi à l'avant-plan le rôle de la motivation autodéterminée dans le fonctionnement optimal au travail.

\section{La théorie d'autodétermination}

Dans le but de comprendre les fondements des problèmes de santé psychologique et de roulement de personnel des intervenants en PJ, il est proposé d'examiner la motivation au travail des intervenants en protection de l'enfance à l'aide de la théorie d'autodétermination (TAD; Deci et Ryan, 1985, 2000; Ryan et Deci, 2017). La TAD fut élaborée aux tournants des années 80 , alors qu'un débat subsistait quant aux effets des récompenses sur la motivation. À cette époque, le courant behavioriste battait son plein et mettait de l'avant l'utilisation de récompenses pour favoriser l'émission des comportements souhaités. Dans le milieu du travail, Porter et Lawler (1968) ont élaboré un modèle de motivation intrinsèque et extrinsèque du travail. En plus de définir la motivation intrinsèque et extrinsèque, ce modèle proposait de bâtir un environnement de travail offrant des récompenses intrinsèques et extrinsèques à la suite d'une performance satisfaisante. Néanmoins, plusieurs 
études ont mis en doute l'efficacité des récompenses pour encourager l'émission de comportements puisque d'une part, les effets des récompenses cessent une fois qu'elles sont retirées et d'autre part, certains comportements sont émis en l'absence de récompenses tangibles (par exemple., Deci, 1971). De fil en aiguille, la théorie de l'évaluation cognitive (CET; Deci, 1975; Deci et Ryan, 1980) a été élaborée afin de mieux expliquer les effets néfastes des récompenses extrinsèques sur la motivation intrinsèque, et mena plus tard au développement de la TAD (Deci et Ryan, 2000; Ryan et Deci, 2017) afin d'expliquer les processus psychologiques sous-jacents de ces effets. Comparativement aux autres théories motivationnelles, la TAD se démarque, car:

elle couvre à la fois les différents types de motivation ([en termes de] qualité) et leur présence ([en termes de] quantité), alors que d'autres modèles ont des catégorisations trop larges (par exemple intrinsèque versus extrinsèque) ou indiquent uniquement des manifestations comportementales au travail sans énoncer les attitudes ou motivations sous-jacentes (Forest, CrevierBraud et Gagné, 2009, p. 25).

Selon la TAD, l'être humain est prédisposé à s'engager dans des activités plaisantes, à utiliser et mettre à profit son plein potentiel, à développer des liens significatifs avec son entourage, et à intégrer ses expériences dans un tout cohérent. Toutefois, l'environnement dans lequel l'individu évolue joue un rôle crucial dans l'atteinte ou non de cette tendance organismique. Autrement dit, il peut soutenir ou contrecarrer l'intériorisation des activités, ainsi que les objectifs et valeurs rattachés à celles-ci, et donc influencer le type de motivation que la personne développera.

La TAD soutient que l'intériorisation des activités est facilitée par la satisfaction des trois besoins psychologiques fondamentaux et universels (Deci et Ryan, 2000), soit l'autonomie, la compétence et l'affiliation sociale. Ceux-ci seraient essentiels au bien-être des individus et à leur développement. Plus précisément, l'autonomie réfère au besoin d'être à l'origine de ses choix et d'agir conformément à ses valeurs. Le besoin de compétence renvoie aux sentiments d'avoir un impact sur son environnement, alors que le besoin d'affiliation sociale traduit le besoin de se sentir respecté et apprécié par son entourage, ainsi que le besoin de respecter et apprécier les gens autour de soi. Les environnements soutenant la satisfaction de ces besoins permettent le développement d'une motivation autodéterminée, alors que les environnements brimant ces besoins limitent, voire empêchent, le processus d'intériorisation et faciliteront l'émergence d'une motivation contrôlée. Ces propositions furent confirmées par plusieurs recherches, notamment celles de Baard, Deci et Ryan (2004) et Laframboise et al. (2016).

Notons que la motivation se définit comme un "construit hypothétique utilisé pour décrire les forces internes et, ou externes produisant le déclenchement, la direction, l'intensité, et la persistance du comportement " (Vallerand et Thill, 1993, p. 18). Tel que mentionné plus haut, la TAD stipule l'existence de deux grands types de motivation, ancrés sur un continuum allant d'une motivation autodéterminée à une motivation contrôlée (Deci et Ryan, 2000). Une motivation autodéterminée est le fruit d'une intériorisation de l'activité dans le soi, de sa valeur et de ce qu'elle représente (Deci et Ryan, 1985). Si l'activité mène à des objectifs 


\section{6}

ou reflète des valeurs qui sont bien intériorisées chez l'individu, une activité qui n'est pas nécessairement plaisante peut être régulée de façon autodéterminée. Ce type de motivation au travail prédit positivement le bien-être psychologique, la satisfaction au travail, l'engagement et la performance (Diefendorff et al., sous presse; Laframboise et al., 2016). Une motivation contrôlée est présente lorsque l'individu exerce l'activité parce qu'il y est obligé ou parce qu'il se sentirait honteux ou coupable s'il ne la faisait pas. Dans ce cas, l'intériorisation est partielle, voire absente. Ainsi, l'activité est perçue comme purement instrumentale. Elle sert à obtenir ou à éviter quelque chose. Ce type de motivation est lié négativement au bien-être psychologique des travailleurs et à la performance, alors qu'il l'est positivement avec l'intention de quitter son emploi (Diefendorff et al., sous presse; Laframboise et al., 2016).

\section{Objectifs de recherche}

Les ressources et les demandes présentes dans un environnement de travail agissent sur le bien-être et l'engagement par l'intermédiaire de leurs effets sur la motivation des travailleurs selon le modèle "Job Demands-Resources ". La description du contexte de travail de la PJ met en lumière un environnement avec des demandes élevées et des ressources limitées, ayant ainsi le potentiel de brimer la satisfaction des besoins psychologiques des intervenants et, par le fait même, affecter leur motivation au travail. L'objectif principal de cette étude est donc d'explorer la motivation au travail en s'intéressant d'abord aux motifs initiaux de carrière (pourquoi avoir choisi de travailler en PJ) et aux sources de motivation (les irritants et les avantages perçus dans l'environnement de travail) des travailleurs québécois en protection de l'enfance.

\section{Méthode}

Les données utilisées pour la présente étude sont issues d'un projet de recherche plus large, à devis mixte (Geoffrion, 2015) portant sur la santé psychologique des intervenants en PJ. Ce projet a reçu l'approbation éthique de I'Université de Montréal et du Centre jeunesse de Québec-Institut Universitaire en novembre 2013.

Des intervenants d'un CISSS en banlieue de Montréal ont été sollicités à la suite de leur participation à un questionnaire en ligne. Compte tenu de leur première participation au volet quantitatif, ceux-ci ont été sollicités à nouveau. Un échantillon de convenance (Miles, Huberman et Saldaña, 2014) a ainsi été assemblé. En fonction de la saturation empirique, 30 entretiens semi-structurés ont été menés auprès de ces intervenants. La saturation empirique est considérée atteinte lorsque le dernier entretien effectué n'apporte plus suffisamment de nouvelles informations pour justifier la poursuite de la collecte de données (Pires, 1997). Par ailleurs, tel que soutenu par Miles et al. (2014), étant donné que nos objectifs de recherche visent l'approfondissement de notre compréhension de la motivation au travail de nos participants, notre échantillon est satisfaisant. 
Ces entretiens téléphoniques, d'une durée moyenne d'une heure, ont été menés à l'aide d'un guide d'entretien comprenant sept thèmes : la motivation au travail, le cheminement professionnel, le travail d'intervenant, l'exposition à la violence, l'exposition au matériel traumatique, l'imputabilité et la culture organisationnelle. Des relances étaient également prévues afin de favoriser l'exploration du contenu pertinent aux questions de recherche par les participants. L'ensemble des entretiens a été enregistré, puis retranscrit. Afin de préserver la confidentialité et l'anonymat des participants, un code a été attribué à chacune des entrevues téléphoniques. Enfin, les participants ont été libérés d'une heure de travail afin de participer à l'étude.

Au moment de l'étude (entre novembre 2013 et juillet 2014), l'ensemble des participants résidait dans la province du Québec et parlait français. Parmi l'échantillon, 16 des répondants étaient éducateurs alors que 14 étaient agents en relation humaine $(\mathrm{ARH})$. Parmi tous les répondants, $60 \%$ étaient des femmes. Les participants étaient âgés en moyenne de 37,6 ans et travaillaient, pour le centre jeunesse, depuis 10,7 années en moyenne.

\section{Stratégie d'analyse}

Une analyse thématique a été menée dans le cadre de cette étude. Selon Paillé et Mucchielli (2016), elle permet de «transposer le corpus en un certain nombre de thèmes représentatifs de l'ensemble du contenu analysé » (p. 236). En plus de permettre le repérage des thèmes importants, ce type d'analyse permet de documenter les liens, les contrastes et les nuances entre les thèmes afin de dresser un portrait d'ensemble des grandes tendances du corpus.

Une analyse thématique séquenciée a donc été effectuée. Dans un premier temps, une partie du corpus (4-5 entrevues) a été analysée en vue de créer une fiche thématique. Celle-ci correspond à une liste de thèmes organisés et définis. Ensuite, cette fiche thématique a été appliquée au reste du corpus. Ce type d'analyse thématique est avantageux, car il « permet une analyse efficace et uniforme » d'un corpus important (Paillé et Mucchielli, 2016, p. 241).

Par la suite, les thèmes liés à la motivation au travail ont été dégagés afin d'arriver à une compréhension riche et approfondie des enjeux entourant la motivation des intervenants rencontrés.

\section{Résultats}

Les résultats sont organisés de manière à préciser les motifs ayant incité les individus à entamer une carrière en PJ (motifs de carrière initiaux), les sources actuelles de motivation dans l'environnement de travail puis les répercussions négatives de ces sources rapportées par les participants. 
Motifs initiaux de carrière : attraits initiaux pour la PJ

Motifs opportunistes. L'analyse des données révèle que plusieurs participants se sont exprimés sur les raisons qui avaient motivé leur choix pour le travail en PJ. En effet, plus de la moitié des participants mentionne des motifs qu'on peut qualifier d'opportunistes. Par exemple, quelques participants rapportent s'être orientés vers la PJ à la suite de diverses circonstances de vie, telles qu'un stage étudiant ou un affichage de poste. Ce sont ces circonstances de vie plutôt que l'intérêt pour l'emploi qui ont motivé le choix de travailler en PJ. Benjamin, 49 ans et éducateur, s'explique son choix d'emploi en ces mots " c'est un accident de parcours ». Pour lui, le choix d'aller en PJ n'était pas un choix longuement réfléchi, mais plutôt un choix de carrière de dernière minute. À la suite d'un stage et de l'obtention d'un diplôme donnant accès à des postes en PJ, Benjamin dit être resté « accroché » au milieu.

Près de cinq participants se sont dirigés en PJ sur la base des nombreuses possibilités d'emploi. Ils disent aimer la diversité des postes, car elle favorise une mobilité du personnel au sein de l'organisation et des occasions d'avancement professionnel. Environ neuf participants disent s'être orientés vers la PJ compte tenu de la réussite de l'entrevue d'embauche et des avantages sociaux. Pour ces participants, c'est le désir d'obtenir un emploi qui apparait comme la principale source de motivation. Pour Roberte, 26 ans et éducatrice, " c'est le Centre jeunesse qui est venu à moi [...]. Ils ont passé des entrevues... Puis j'ai appliqué, j'ai été acceptée ». Ainsi, avoir été retenu par l'organisation a suffi à convaincre certains individus d'entreprendre une carrière en PJ. Huit participants mentionnent que le fait de connaitre des personnes dans le milieu de la PJ, tel qu'un ami, les a incités à s'orienter vers ce type d'organisation. Les propos de Valérie, 23 ans et éducatrice, s'inscrivent dans cette continuité :

« C'est un métier, c'est un milieu de travail qui avait été valorisé beaucoup dans ma [formation] technique [au Cégep], parce que j'avais plusieurs enseignants qui ont travaillé là. J'ai un ami aussi qui a travaillé là avant moi, qui aimait beaucoup son travail. Puis, j'ai appliqué. »

Motifs relationnels. Près de 25 intervenants ont expliqué ce choix par des motifs relationnels comme un intérêt pour la relation d'aide et le plaisir à l'exercer. Parmi ceux-ci, certains participants rapportent avoir choisi le travail en PJ, car ils aiment beaucoup la clientèle juvénile ou la clientèle familiale. Stéphanie, 26 ans et éducatrice, rapporte en entretien que " ce n'était pas tant le contexte d'autorité qui me motivait que la proximité avec les jeunes ». D'autres participants rapportent être allés en $\mathrm{PJ}$ pour faire la différence auprès de la clientèle. Beaucoup de participants souhaitaient contribuer à la vie des jeunes en la modifiant positivement. Plus précisément, quelques participants disent avoir été en PJ, car ils souhaitaient aider les jeunes. Arnaud, 27 ans et éducateur, mentionne que c'est « la relation d'aide qui m'intéresse ».

Motifs personnels. Le fait de s'identifier personnellement au rôle d'intervenant ainsi que les expériences antérieures ont alimenté le désir de près du tiers des participants de travailler en PJ... Simon, 41 ans et éducateur, exprime cette 
idée en mentionnant avoir choisi le travail en PJ pour des « raisons familiales... nous avons trempé là-dedans... mon père était en famille d'accueil pendant sept ans ». Cette histoire de vie a, en partie, façonné la personnalité de l'intervenant et son intérêt pour la relation d'aide auprès des jeunes.

Pour certains participants, le choix de travailler en PJ repose sur la perception de sa propre identité personnelle. L'individu reconnait dans son caractère ou sa personnalité, une tendance naturelle à aider autrui. Les propos d'Émilie, 26 ans et éducatrice, l'illustrent adéquatement : "j'ai toujours été quelqu'un de sociable, qui aime les gens [...]. Puis j'ai toujours aimé prendre soin des autres, écouter les autres, conseiller. Donc, ça allait un peu dans cette même lignée-là ».

Motifs liés aux particularités de la PJ. Ces motifs représentent des éléments caractérisant le travail en protection de l'enfance, dont les problèmes rencontrés (par exemple., la délinquance juvénile). Environ un tiers des participants se disent intéressés à travailler en PJ à cause des spécificités du milieu. C'est le cas de Patrice, 30 ans et éducateur, qui dit aimer « les cas un peu plus problématiques au niveau des jeunes, de comment les prendre, comment les twister, comment essayer de trouver une façon de les motiver, puis de les amener à être fonctionnel en groupe " ou de Jean-François, 26 ans et ARH, qui trouve "que le Centre jeunesse, ça offre de la variété au niveau de la nature des problèmes ». Pour certains participants, la perspective d'avoir d'importants défis cliniques est attrayante. La présence de dossiers complexes et variés apparait intéressante. Anne mentionne que « je voulais changer de milieu. Je voulais de nouveaux défis ».

Ces résultats suggèrent que, pour les intervenants rencontrés, les motifs ayant incité les individus à entamer une carrière en PJ, sont nombreux et variés. De plus, la plupart des motifs évoqués sont de nature autodéterminée, c'est-à-dire qu'ils réfèrent à des caractéristiques de la personne (par exemple., tempérament), à des valeurs (par exemple., altruisme), des croyances (par exemple., croire en la nécessité d'aider les personnes démunies) ou des intérêts (par exemple., s'intéresser aux problématiques juvéniles). Seuls les motifs opportunistes rappellent une motivation de type contrôlée, c'est-à-dire que la personne concernée affirme agir en vue d'obtenir une récompense, dans le cas présent, un emploi ou éviter une situation désagréable.

\section{Sources de motivation au travail : motifs de carrière actuels}

La Figure 1 résume les sources de motivation au travail, en distinguant les irritants et les avantages perçus dans l'environnement de travail.

\section{Les irritants du travail en PJ}

De façon plus détaillée, les participants se sont prononcés sur les irritants qu'ils percevaient dans leur environnement de travail en PJ. À cet effet, la section suivante présente ce qui est perçu comme irritant dans l'environnement de travail en $\mathrm{PJ}$ à différents niveaux. 

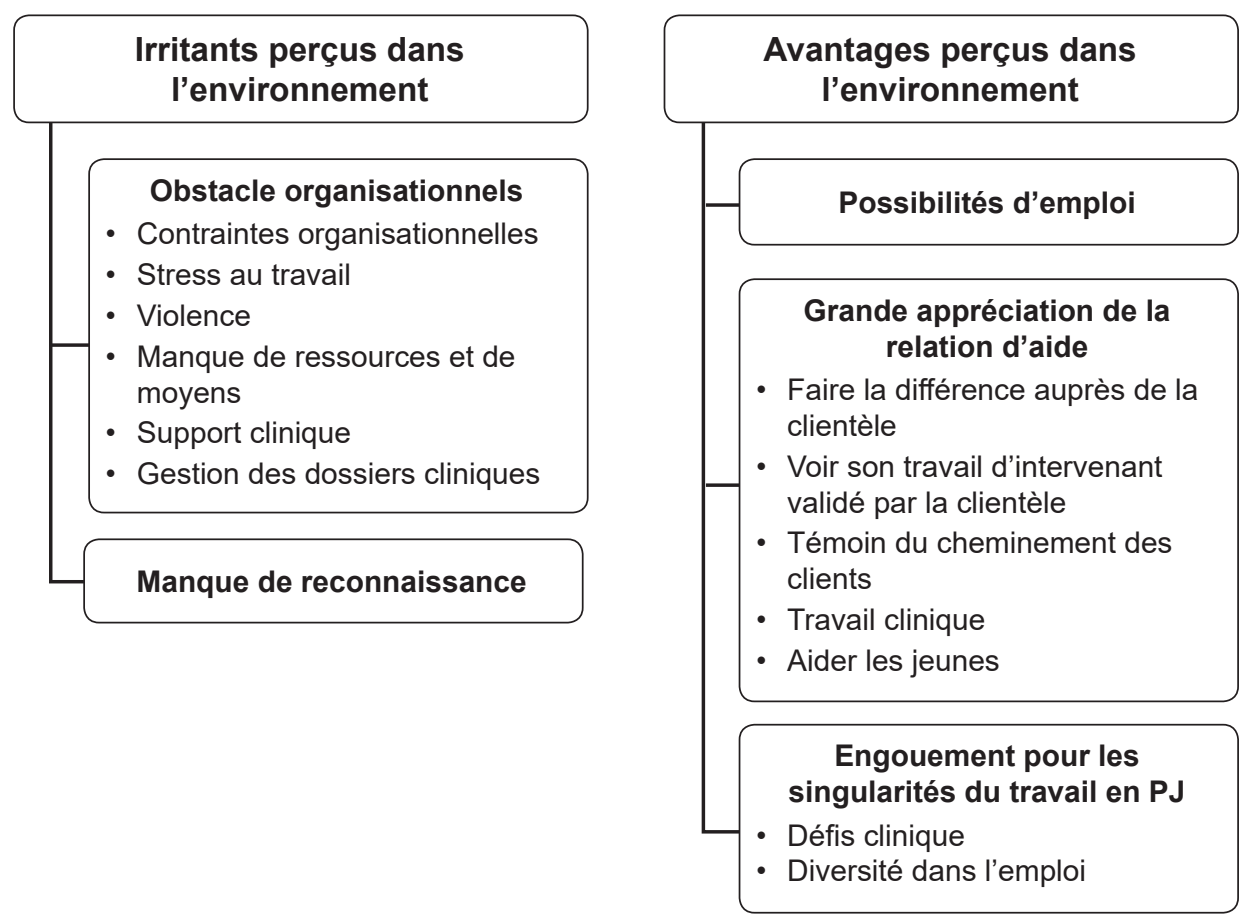

Figure 1. Résumé des principales sources de motivation au travail.

Obstacles organisationnels. Moins du tiers des participants trouvent difficile la présence d'obstacles organisationnels, de limites et de contraintes dans leur environnement de travail. Pour Mathieu, 53 ans et ARH, les limites provoquent "une lourdeur». Anne explique que "ce que j'aime moins, c'est justement le contexte d'autorité qui, des fois, peut nous mettre des grandes limites ». Les contraintes organisationnelles comprennent, notamment, l'ensemble des procédures et des règlements régissant le travail des intervenants. Elles sont perçues par les travailleurs comme astreignantes et pénibles.

Trois participants disent peu apprécier l'important niveau de stress vécu au travail. Mathieu explique :

"Quand qu'on parle du stress, c'est [en raison du] nombre de dossiers. Ça fait des années et des années, mais qu'est-ce que tu veux ça c'est une contrainte qui ne pourra jamais se changer parce qu'on a des quotas puis on a des choses du ministère [...] c'est un job de fou que nous avons. "

Plusieurs éléments, dont la surcharge de travail et le contexte d'autorité, contribuent au stress vécu par les participants. L'extrait précédent expose l'important sentiment d'impuissance de certains participants face aux stresseurs perçus négativement dans l'environnement de travail. 
Quatre participants ont expliqué vivre difficilement avec l'omniprésence de la violence en PJ. Romy, 39 ans et éducatrice, explique « qu'il faut toujours que tu aies en tête que lui, il a un potentiel d'agir, il peut t'agresser n'importe quand [...] ça vient lourd à porter $»$. Cet extrait illustre que la violence est certes vue comme un agent démotivant, mais elle influence aussi les comportements et les attitudes au travail, par exemple le développement de préoccupations et la méfiance.

Le manque de ressources et de moyens afin d'accomplir adéquatement leur travail est perçu comme décourageant par trois participants. Être confronté à beaucoup de demandes, d'exigences et de responsabilités en ayant peu de moyens pour y répondre occasionne des frustrations chez certains, dont Roberte qui mentionne que «c'est très difficile sur le moral vu les manques de ressources qu'on a aussi là ». Cette citation expose la difficulté d'avoir un sentiment de compétence, d'efficacité dans un tel contexte de travail.

D'après quelques participants, le support clinique offert en PJ est perçu comme frustrant et démotivant. À titre d'exemple, Benjamin dit «au lieu du support clinique, on nous encadre, tu n'as pas de marge de manœuvre, ça devient démotivant». Mathieu explique que ses supérieurs " sont toujours en train de me dire ben tes P.I. sont pas à jour, tes ci à jour... ». Ces citations exposent le peu d'espace consacré au soutien émotionnel des employés. Elles illustrent également la faible marge de manœuvre dont bénéficient les intervenants en PJ et la difficulté de faire des choix correspondant à leurs valeurs.

Par ailleurs, pour près de deux de nos participants, la gestion des dossiers cliniques est perçue comme problématique. Ils disent avoir de la difficulté à travailler de manière efficace auprès de la clientèle compte tenu de l'orientation des plans d'intervention et de la rotation des dossiers. C'est le cas de Lou, 37 ans et éducatrice, qui rapporte "qu'on se concentre juste sur un symptôme. Le jeune est en réaction, il a de mauvais comportements. Mais ça, c'est un symptôme de quelque chose d'autre ". Anne, quant à elle, dit être découragée davantage par le fait de devoir dire "bien votre suivi commence, mais dans un mois, ça ne sera plus moi qui serai là. Ça va être une autre personne ». Anne ajoute que " c'est difficile de créer le lien, de les mettre en confiance, d'essayer d'installer le plan d'intervention ».

Manque de reconnaissance. Le manque de reconnaissance du travail effectué par la population ou par la clientèle est perçu comme frustrant et démoralisant par deux participants. À titre d'exemple, Anne dit « notre travail n'est pas nécessairement reconnu, nous sommes vite stigmatisés à la méchante DPJ, les voleurs d'enfants. Ce côté-là, il est plus difficile [...] ». Cette citation indique que le besoin d'affiliation de la participante n'est pas comblé, car l'intervenante ne se sent pas appréciée et reconnue par ceux qui l'entourent.

\section{Répercussions négatives rapportées par les participants}

Lors de l'analyse, les participants ont également décrit des répercussions négatives face aux éléments irritants de leur environnement de travail en PJ. La Figure 2 illustre les répercussions négatives et positives perçues 


\section{Répercussions négatives perçues}

- Quitter son poste ou la PJ

- Déplacer son intérêt pour l'emploi vers les conditions de travail

- Désillusion face à son emploi ou son organisation

- Diminution de l'investissement au travail

\section{Répercussions positives perçues}

- Appréciation du mandat de travail

- Augmentation de l'investissement au travail

- Demeurer en poste ou en PJ

\section{Figure 2. Répercussions négatives et positives perçues.}

Joëlle, 30 ans et éducatrice, dit que certains éléments dans l'environnement de la PJ affectent ses « plans de carrière ». Cette participante ajoute que :

"Ça fait plusieurs années que j'en parle à ma chef de service que je veux me réorienter, et ce, pour diverses raisons, mais beaucoup à cause de la violence, de la surcharge de travail, du peu de moyens qui nous est offert. À long terme, je ne peux pas garantir que je vais être encore dans le Centre jeunesse ».

Les propos de Roberte s'inscrivent en continuité aux propos précédents. Elle affirme : " à long terme, je me vois peut-être travailler dans un CLSC ». Compte tenu d'un ensemble de sources de frustration, telles que l'horaire de travail ou la violence, près d'un tiers des participants disent vouloir quitter un jour leur poste ou la PJ.

Trois des participants disent avoir déplacé leur intérêt pour l'emploi vers les conditions de travail de celui-ci. Plutôt que de travailler par plaisir, certains participants demeurent en poste afin de continuer à bénéficier des avantages sociaux offerts par l'employeur tels que le régime de retraite. Les propos de Lou appuient cette idée lorsqu'elle explique : " si je pouvais travailler avec les mêmes jeunes en étant travailleuse de rue pour le même salaire, les mêmes conditions de travail, j'opterais pour ça à cent mille à l'heure ». De plus, Mathieu mentionne honnêtement en entretien : " je reste pour la pension ».

Trois intervenants rapportent être désillusionnés face à leur emploi ou à l'organisation. Ils mentionnent un important décalage entre la façon dont ils percevaient leur travail au début de leur carrière et comment ils le perçoivent au moment de l'entretien. Lou déclare :

" J'ai tellement rêvé de ce job-là... Je m'en allais dans quelque chose que j'aimais, une clientèle que j'aimais. J'avais beaucoup de rêves, beaucoup d'espoirs, puis je suis comme tombée de haut. C'est sûr que ce n'est 
pas évident de travailler quand tu ne crois pas au système puis j'ai eu des difficultés avec mes collègues, ma chef d'unité [...]. Je suis comme désillusionnée de ma job. »

Benjamin dit plutôt de son organisation et de son travail que " ça a été avec le temps comme une vocation, qui est devenue aujourd'hui plus comme une occupation ». Pour ces participants, le travail était autrefois perçu comme une source de stimulations, d'attentes, de rêves, d'espoirs et de satisfaction. II est désormais perçu comme une source de frustrations, d'insatisfactions alimentant des sentiments de résignation et d'impuissance.

Mathieu a affirmé avoir observé, au fil du temps, une évolution quant à son niveau d'investissement au travail. Après 30 années en PJ, cet employé dit " avant là, j'étais sur les tables de ci puis de ça pour faire des discussions puis changer. Aujourd'hui, ils voudraient bien que je fasse des affaires, que j'utilise mon expérience, bien non! » Le comportement de Mathieu et son attitude envers l'organisation démontrent un certain désengagement de sa part ainsi qu'un refus à s'investir davantage au travail.

\section{Avantages perçus dans l'environnement de travail}

Plusieurs possibilités d'emplois. Six des participants à l'étude se disent enthousiasmés par les nombreuses occasions de carrière en PJ. La possibilité de changer d'emploi en demeurant dans l'organisation apparait comme un avantage pour certains intervenants. Par exemple, Stéphanie dit qu'elle ne sait pas quel poste elle occupera à l'avenir, mais que son plan de carrière «c'est les Centres jeunesse jusqu'à ma retraite ». Nicolas, 35 ans et éducateur, dit "ce que j'aimerais, c'est devenir conseiller au développement professionnel ».

Grande appréciation de la relation d'aide. Près du trois quarts des intervenants disent aimer des éléments liés à la relation d'aide en protection de l'enfance. Pour près d'un tiers des participants, l'impression de faire la différence auprès de la clientèle est perçue comme une source de stimulation, de plaisir et de motivation au travail. Alice, 47 ans et $\mathrm{ARH}$, dit apprécier « savoir que je peux à un moment donné faire oublier que je suis en contexte d'autorité puis juste faire une petite différence, les amener ailleurs, être là pour eux autres ». Cette citation illustre aussi qu'un travailleur qui perçoit faire la différence auprès de sa clientèle est un intervenant dont le besoin de compétence est satisfait.

Cinq intervenants disent aimer voir leur travail d'intervenant validé par la clientèle. Ils rapportent adorer lorsque les jeunes reviennent les voir pour les remercier, les saluer, les informer de leur cheminement. Pour Gabrielle, 27 ans et éducatrice, "le plus beau du travail », c'est carrément "quand un ancien jeune revient pour nous remercier de ce qu'on a pu faire pour lui ». Mathieu seconde en disant que voir un jeune revenir quelques années plus tard avec de bonnes nouvelles, c'est ça son "trophée ». Ces extraits d'entretiens indiquent que les intervenants se sentent gratifiés, fiers et compétents lorsqu'ils voient leur travail d'aidant validé par la clientèle. 
Six des sujets à l'étude disent être mobilisés par le fait d'être témoin du cheminement des clients. Pour Monique, 34 ans et $\mathrm{ARH}$, il est plaisant « de voir les petites réussites... de voir qu'ils sont capables de cheminer même si des fois, ce n'est pas des grands pas ». Pour d'autres, comme Simon, il est inspirant d'être témoin de la résilience des clients. En entrevue, cet éducateur dit « ça, c'est ce que j'apprécie dans ce travail, c'est de voir la capacité des jeunes qui, malgré un paquet de facteurs, sont capables de réfléchir puis de grandir au travers de toute leur vie ».

Quelques intervenants ont expliqué que le travail clinique est un important incitatif au travail. Émilie dit aimer « le relationnel avec les jeunes, leur parler, faire des activités en contexte plus ludiques comme quand qu'on fait des activités plus sportives ou des jeux de table ou des trucs comme ça ». Une grande appréciation pour le volet clinique se dégage de plusieurs entretiens. Les intervenants ont rapporté apprécier la relation d'aide, le contact direct avec la clientèle ou le fait de discuter de cas cliniques.

Pour plus de la moitié des participants, le fait d'aider les jeunes représente la principale source de motivation. À titre d'exemple, la relation d'aide est un élément du travail en protection de l'enfance grandement apprécié. Rosalie, 29 ans et ARH, explique qu'elle aime "beaucoup travailler avec les enfants [...], les parents puis avec ce que ça amène comme dynamique... en se centrant toujours sur le besoin des enfants ». La relation d'aide est perçue, pour plusieurs, comme une expérience " enrichissante ", " ressourçante » et une source de " gratification », de " plaisir » et de valorisation, bref comme une tâche ayant un sens.

Engouement pour les singularités du travail en PJ. Près du tiers des participants disent aimer certaines particularités du travail en protection de l'enfance. Spécifiquement, pour plusieurs participants, considèrent la présence de défis cliniques comme un facteur mobilisant. Le témoignage de Nicolas s'inscrit dans cette perspective «tu as deux cas, tu n'as pas deux personnes pareilles, tu n'as pas justement deux jeunes qui vont réagir exactement de la même façon à la même intervention. C'est tout ce petit défi qui est tout sauf monotone ». Valérie dit « au début, c'était vraiment une réalité que je ne connaissais pas. Alors, il y a ça aussi que j'ai trouvé super stimulant ». Pour ces intervenants, les défis cliniques provenant de la clientèle, des situations rencontrées et du contexte de la relation d'aide sont vécus comme stimulants. Ils permettent à certains, dont Valérie, de sortir de leur « zone de confort » au travail.

Pour cinq participants, la grande diversité dans l'emploi est un incitatif au travail. Cette diversité découle, notamment, de la variété des problématiques rencontrées, de la clientèle et de l'environnement de travail (par exemple., bureau, foyer familial). À cet effet, Maéva, 43 ans et $\mathrm{ARH}$, dit de son travail que « ce n'est pas un travail routinier, c'est diversifié, on ne voit jamais le même monde ».

\section{Répercussions positives rapportées par les participants}

Les répercussions positives face aux avantages perçus dans l'environnement ont émergé lors de l'analyse. Celles-ci génèrent, chez un peu moins du tiers des participants, une importante appréciation de leur mandat de travail. Pour un 
résumé des répercussions positives décrites par les sujets, le lecteur est invité à consulter la Figure 2. Amir, 41 ans et $\mathrm{ARH}$, dit " j'aime le mélange d'être sur le terrain puis d'être au bureau. J'aime les formations. J'aime aider les gens. J'aime voir le sourire d'un jeune que ça fait longtemps que tu n'as pas vu sourire. C'est les petits moments comme ça qui te font continuer là ». L'analyse démontre que ces participants sont passionnés par leur emploi et qu'ils disent retirer beaucoup de plaisir à travailler en PJ. Ce qu'ils disent aimer dans leur mandat de travail ce sont, entre autres éléments, la relation d'aide et les problèmes rencontrés par la clientèle. Sylvain, 45 ans et $\mathrm{ARH}$, illustre bien son amour pour son emploi en disant avoir refusé dans sa carrière d'autres opportunités d'emploi alléchantes. Cet intervenant rapporte que "c'est un privilège de faire quelque chose que j'aime pis pour lesquelles, à tous les matins, jamais jamais je rentre à reculons ».

Quatre des participants affirment que le fait d'être plus investis au travail représente une répercussion positive découlant des modalités appréciées dans l'environnement de la PJ. Pour Arnaud, le travail « ça va plus loin » que les quarts de travail. Cet intervenant dit "j'organise mes activités sur mon temps à moi des fois. Je prépare des choses pour mes gars en dehors de mon temps souvent. Mais c'est quelque chose qui me convient ». Selon cet extrait et d'autres, l'investissement se mesure par le travail effectué à l'extérieur du boulot, par le temps passé en fonction ou encore par l'attachement des intervenants à la clientèle. Ces participants disent s'investir beaucoup au travail, car ils y trouvent un sens.

Un tiers des intervenants ont mentionné désirer demeurer au sein de l'organisation ou garder leur poste. Simon explique : " je pense que je suis encore bien dans ce que je fais, et tant que je me sens bien dans ce que je fais, je n'ai pas besoin de me voir ailleurs ». Cet extrait illustre l'importance du bien-être de l'employé dans le choix de demeurer en poste.

\section{Discussion}

L'objectif de cette étude était d'explorer la motivation au travail. Les résultats obtenus à l'aide d'un devis qualitatif offrent une meilleure compréhension de l'expérience motivationnelle des intervenants en PJ du Québec dans la mesure où il jette un éclairage sur les motifs à initier une carrière en $\mathrm{PJ}$ et les sources motivationnelles actuelles. Les participants ont également partagé la perception qu'ils avaient des répercussions négatives et positives des sources motivationnelles actuelles. Les participants ont ainsi offert une analyse de leur milieu de travail et de leur emploi en mentionnant les sources actuelles de motivation, soit les irritants et les avantages perçus dans l'environnement de travail. Selon le modèle de « Job Demands-Resources » (Bakker, Demerouti et al., 2003; Bakker, Demerouti, Taris et al., 2003; Demerouti, et al., 2000, 2001), ces éléments peuvent correspondre aux demandes et aux ressources liées au travail d'intervenant en PJ. De plus, les résultats de l'étude appuient une des prémisses de la TAD (Deci et Ryan, 1985, 2000), soit que la motivation au travail est un processus dynamique variant selon l'individu et l'environnement de travail.

À la lumière des analyses, la plupart des participants se disaient intéressés par la PJ pour des motifs de carrière initiaux de type opportuniste et relationnel. 


\section{6}

Comme le suggère la TAD (Deci et Ryan, 1985, 2000), la motivation au travail des intervenants en PJ est composée de motifs initiaux de carrière ayant des niveaux d'autodétermination différents. En plus d'être motivés par le fait d'avoir un emploi et de bons avantages sociaux, les intervenants en PJ disent initier leur carrière afin de se réaliser au quotidien en aidant autrui ou d'avoir du plaisir en aidant les jeunes en difficulté.

Plusieurs études évoquent que les individus présentant une motivation au travail autodéterminée affichent un niveau de bien-être psychologique supérieur (par exemple., Fernet et al., 2012; Nie et al., 2014; Trépanier et al., 2013) et rapportent vivre davantage d'émotions positives (par exemple., Nix, Ryan, Manly et Deci, 1999). Richer, Blanchard et Vallerand (2002) proposent que la motivation de type autodéterminée soit associée à moins d'épuisement émotionnel et à plus de satisfaction au travail chez les employés. D’autres études (par exemple., Gagné, Chemolli, Forest et Koestner, 2008; Gillet, Huart, Colombat et Fourquereau, 2013; Van den Broeck, Lens, De Witte et Van Coillie, 2013) suggèrent qu'une motivation autodéterminée est associée à un niveau plus élevé d'engagement au travail. Les conclusions des travaux de Gillet, Gagné et al. (2013) ainsi que celles d'Otis et Pelletier (2005) suggèrent que plus la motivation au travail est autodéterminée, plus les travailleurs rapportent l'intention de rester dans leur emploi. À première vue, il existe une inconsistance entre ces résultats et les données issues de la présente étude. Malgré une motivation initiale au travail en grande partie autodéterminée, quelques participants rapportent des répercussions en emploi, certains affirment même vouloir le quitter. Il est plausible que malgré une motivation initiale autodéterminée envers le travail d'intervenant en PJ, le milieu de travail, et plus particulièrement les demandes et les ressources disponibles en PJ, aient contribué à moduler la motivation actuelle des participants. En effet, les participants rapportent faire un travail à demandes élevées, dans un contexte où les ressources sont limitées. Selon l'étude de Fernet et collègues (2012), ce type de contexte influencerait négativement la motivation autodéterminée au travail, ce qui en retour faciliterait l'émergence de problèmes de santé psychologique, d'engagement et de roulement de personnel.

Dans le but d'explorer cette hypothèse, les intervenants ont été invités à partager les irritants et les avantages perçus dans l'environnement de travail de la PJ, soit des éléments se rapportant aux demandes et ressources de leur travail. L'analyse des propos indique que les obstacles organisationnels sont nommés de façon récurrente lorsque les participants se prononcent sur ce qui les irrite ou ce qu'ils n'aiment pas dans l'environnement de la PJ. Güntert (2015) décrit les facteurs organisationnels comme les plus importants et les plus étudiés afin d'expliquer la motivation au travail. D'autres études (par exemple., Ellet et al., 2007; Martinez, 2004) démontrent la contribution notable de ces facteurs au bien-être des employés en PJ et au taux de roulement.

Suivant ces résultats, il est proposé que les irritants perçus par les intervenants dans l'environnement de travail ne permettent pas, en réalité, la satisfaction des besoins psychologiques et génèrent conséquemment une motivation davantage contrôlée chez ceux-ci. Rappelons que selon la TAD (Deci et Ryan, 1985, 2000), une motivation contrôlée émerge lorsque l'individu exerce 
une activité qu'il n'aime pas ou qu'il perçoit comme déplaisante ou non conforme à ses valeurs. Dans un tel cas, l'intériorisation de l'activité est partielle ou absente puisque les besoins psychologiques sont frustrés. Nos résultats suggèrent que les demandes trop élevées ainsi que le manque de ressources reliées au travail en PJ réduisent la qualité de la motivation au travail des intervenants, par l'entremise de la réponse partielle aux besoins psychologiques, et, conséquemment, engendrent des problèmes de santé psychologique et de roulement de personnel.

Par ailleurs, l'examen des discours des intervenants indique que ce qui est le plus apprécié dans l'environnement de la protection de l'enfance est la relation d'aide. Selon Deci et Ryan (1985, 2000), une motivation autodéterminée se manifeste quand le travailleur exerce une activité qu'il aime ou encore qu'il perçoit comme plaisante ou conforme à ses valeurs. Pour ces auteurs, l'activité est intériorisée chez le travailleur, car les besoins psychologiques sont satisfaits. En ce sens, la relation d'aide est une activité qui aurait le potentiel de satisfaire les besoins psychologiques des intervenants et de créer une motivation plus autodéterminée. Toutefois, malgré un certain niveau de satisfaction des besoins psychologiques fondamentaux des intervenants en PJ, la présente recherche démontre que l'environnement de travail en PJ limite la plupart du temps l'accomplissement de la relation d'aide. Autrement dit, les contraintes organisationnelles, la violence, le manque de ressources et le faible support clinique font obstacle à la relation d'aide, activité prisée par la majorité des intervenants.

Plus spécifiquement, l'analyse des propos des participants témoigne d'une gestion très hiérarchique, laissant peu de marge de manœuvre aux intervenants. Ce type de gestion favorise peu le soutien à l'autonomie des employés. Or, Baard et collègues (2004), dans une étude réalisée en institution bancaire, ont démontré que les gestionnaires adoptant un style de gestion soutenant l'autonomie (par opposition à ceux adoptant un style de gestion plus contrôlant) ont des employés présentant des niveaux supérieurs de rendement, d'engagement et de bien-être. Pour la TAD, le soutien à l'autonomie perçu est l'élément principal permettant de satisfaire les besoins psychologiques fondamentaux. En ce sens, les recherches (Baard et al., 2004; Deci et al., 2001; Gagné et Deci, 2005) suggèrent que les organisations et les superviseurs peuvent soutenir l'autonomie de leurs employés en instaurant diverses stratégies telles que de permettre à l'employé une latitude lors de la réalisation des tâches, d'expliquer les raisons pour lesquelles une tâche doit être faite, d'offrir des choix significatifs, de considérer l'expérience (p. ex., sentiments, perceptions) des employés et de donner de la rétroaction constructive dans un langage non contrôlant (Diefendorff et al., sous presse).

Outre le style de gestion, l'étude des propos des participants suggère que plusieurs tâches et conditions de travail en PJ ne permettent pas de soutenir leur autonomie. L'analyse des entretiens révèle une importante diversité de clientèles, de tâches, de problèmes et de défis. Toutefois, il semble que les conditions dans lesquelles les intervenants travaillent viennent contrecarrer les possibles bienfaits de cette importante diversité. Par exemple, la violence rencontrée en milieu de travail peut accroitre l'insécurité chez l'intervenant et diminuer ainsi la satisfaction de son besoin de compétence lorsqu'il aide sa clientèle. L'importance de concevoir des tâches et des conditions de travail facilitant la satisfaction des besoins 
psychologiques fondamentaux des employés a pourtant été démontrée dans l'étude de Güntert (2015). Pour ce faire, il est nécessaire de concevoir des postes incluant une certaine autonomie et des tâches diversifiées permettant aux employés d'agir en cohérence avec leurs valeurs personnelles.

Güntert (2015) ajoute qu'il importe de donner une signification au travail (surtout lorsque les tâches effectuées sont difficiles, donc dans un contexte de demandes élevées), en expliquant clairement par exemple comment la réalisation de ces tâches, le respect de certains règlements ou les contraintes législatives permettent d'atteindre les objectifs organisationnels, de contribuer au mieux-être de la population ou de la clientèle desservie ou d'aider les autres employés dans la réalisation de leur propre travail. Les discours des intervenants en PJ suggèrent cependant qu'il est difficile pour ces derniers de saisir le sens derrière certaines tâches ou règlementations. Effectivement, les résultats illustrent que ce sont les contraintes et les procédures organisationnelles plutôt que le sens qui dictent le travail à effectuer.

\section{Implications cliniques}

Conformément aux conclusions des travaux de Baard et collègues (2004), Fernet et collègues (2012) ainsi que de Güntert (2015), il apparait important d'élaborer un environnement de travail en PJ supportant davantage le travail d'intervention auprès de la clientèle en offrant des ressources afin de faire face aux demandes du milieu. Nos résultats suggèrent que cela pourrait permettre une plus grande satisfaction des besoins psychologiques chez les intervenants. De manière intéressante, les résultats de l'étude de Turcotte et Pouliot (2006) avaient proposé que des facteurs organisationnels, comme le soutien organisationnel et l'autonomie au travail, aient une influence significative sur la satisfaction au travail des intervenants en protection de l'enfance. L'étude de Güntert démontre clairement qu'il est en effet plus avantageux pour l'organisation de soutenir les besoins psychologiques des travailleurs puisque cela favorise la motivation autodéterminée et plusieurs conséquences positives. Pour ce faire, il est important d'offrir aux intervenants de la rétroaction constructive (Diefendorff et al., sous presse), de la latitude lors de la réalisation des tâches, de la considération pour leur expérience, des explications sur le sens d'une tâche, des conditions organisationnelles permettant d'investir davantage dans la relation d'aide (par exemple., réduire la quantité de dossiers par intervenant, limiter la rotation des dossiers) (Baard et al., 2004; Deci et al., 2001; Gagné et Deci, 2005). Conformément aux conclusions des études de Gillet, Gagné et al. (2013), d'Otis et Pelletier (2005) ainsi que celles de Trépanier et al. (2013), ces actions permettraient de limiter les problèmes de santé psychologique et de rotation de personnel.

\section{Limites et contributions}

Bien qu'il soit impossible de généraliser ces résultats à l'ensemble des intervenants, il est envisageable de les transférer à une population ou à un contexte similaire (Fortin et Gagnon, 2016). Précisons aussi qu'un biais de sélection ne peut être complètement écarté. Puisque les entrevues se sont déroulées dans le cadre d'un projet de recherche portant sur la violence au travail, il est possible que 
les sujets voulant participer à l'étude fussent ceux qui étaient insatisfaits de leur environnement de travail. Ajoutons que la présente étude ne permet pas de cerner précisément comment la grande appréciation de la relation d'aide alimente la motivation au travail. Aussi, il reste à démontrer clairement comment les obstacles organisationnels dans l'environnement de travail affectent l'évolution de la motivation au travail des intervenants et la prestation de services auprès de la clientèle.

Nonobstant ces limites, la principale contribution de cette étude réside dans le fait que les résultats s'inscrivent en continuité avec les conclusions de travaux actuels (par exemple., Baard et collègues, 2004; Fernet et al., 2012; Gillet, Huart et al., 2013; Güntert, 2015; Otis et Pelletier, 2005). De plus, cette étude montre que l'appréciation de la relation d'aide par les intervenants en PJ a le potentiel de contribuer grandement à la satisfaction de leurs besoins psychologiques et à leur motivation au travail. Toutefois, il semble que les obstacles organisationnels dans l'environnement de travail en PJ entravent la plupart du temps la relation d'aide et, conséquemment, la satisfaction des besoins psychologiques des intervenants.

En somme, cette étude, réalisée auprès de 30 intervenants en $\mathrm{PJ}$, a permis d'explorer la motivation au travail en s'intéressant aux motifs de carrière initiaux (pourquoi avoir choisi de travailler en PJ) et aux sources de motivation actuelles (les irritants et les avantages perçus dans l'environnement de travail) des travailleurs québécois en protection de l'enfance. La présente étude illustre l'importance d'offrir des ressources aux intervenants en développant un environnement de travail en PJ ne portant plus obstacle à la relation d'aide. Cela permettrait de mieux soutenir le bien-être et le désir de demeurer en poste des intervenants en satisfaisant leurs besoins psychologiques fondamentaux et en générant une motivation plus autodéterminée. Autrement dit, sur la base de l'analyse de nos résultats, nous présumons que favoriser un environnement de travail soutenant la relation d'aide aurait le potentiel de protéger les intervenants contre l'adversité présente dans l'environnement de travail en PJ.

\section{Références}

Anderson, D.G. (2000). Coping strategies and burnout among veteran child protection workers. Child Abuse \& Neglect, 24(6), 839-848.

Baard, P.P., Deci, L.E. et Ryan, M.R. (2004). Intrinsic need satisfaction: A motivational basis of performance and well-being in two work settings. Journal of Applied Social Psychology, 34(10), 20452068. doi: 10.1111/j.1559-1816.2004. tb02690.x

Bakker, A.B., Demerouti, E., De Boer, E. et Schaufeli, W.B. (2003). Job demands and job resources as predictors of absence duration and frequency. Journal of Vocational Behavior, 62, 341-356.
Bakker, A.B., Demerouti, E., Taris, T.W., Schaufeli, W.B. et Schreurs, P.J.G. (2003). A multigroup analysis of the job demands-resources model in four home care organizations. International Journal of Stress Management, 10(1), 16-38.

Bell, H., Kulkarni, S. et Dalton, L. (2001). Organizational prevention of vicarious trauma. Families in Society: The Journal of Contemporary Human Services, 84(4), 463-470.

Bennett, S., Plint, A. et Clifford, T. (2005). Burnout, psychological morbidity, job satisfaction, and stress: A survey of Canadian hospital based child protection professionals. Archives Disease in Childhood, 90(11), 1112-1116. 
Deci, E.L. (1971). Effects of externally mediated rewards on intrinsic motivation. Journal of Personality and Social Psychology, 18, 105-115.

Deci, E.L. (1975). Intrinsic motivation. New York, NY: Plenum.

Deci, E.L. et Ryan, R.M. (1980). The empirical exploration of intrinsic motivational processes. Dans L. Berkowitz (dir.), Advances in experimental social psychology (Vol. 13, p. 39-80). New York, NY: Academic Press.

Deci, L.E. et Ryan, M.R. (1985). The general causality orientations scale: Selfdetermination in personality. Journal of Research in Personality, 19, 109-134.

Deci, L.E. et Ryan, M.R. (2000). Selfdetermination theory and the facilitation of intrinsic motivation, social development, and well-being. American Psychologist, 55(1), 68-78. doi: 10.1037110003-066X.55.1.68

Deci, L.E., Ryan, M.R., Gagné, M., Leone, D.R., Usunov, J. et Kornazheva, B.P. (2001). Need satisfaction, motivation, and well-being in the work organizations of a former eastern bloc country. Personality and Social Psychology Bulletin, 27, 930-942.

Demerouti, E., Bakker, A.B., Nachreiner, F. et Schaufeli, W.B. (2000). A model of burnout and life satisfaction amongst nurses. Journal of Advanced Nursing, 32(2), 454-464.

Demerouti, E., Bakker, A.B., Nachreiner, F. et Schaufeli, W.B. (2001). The job demands resources model of burnout. Journal of Applied Psychology, 86, 499512.

Diefendorff, J.M., Houlfort, N., Vallerand, R.J. et Krantz, D.S. (sous presse). Emphasizing the self in organizational research on self-determination theory. Dans J. Russell, C. Sedikides et L. Ferris. (dir.), The self at work. SIOP Organizational Frontiers Book Series. New York, NY: Routledge, Taylor \& Francis Group.
Drake, B. et Yadama, N.G. (1996). A structural equation model of burnout and job exit among child protective services workers. Social Work Research, 20(3), 179-187, doi: 10.1093/swr/20.3.179

Ellet, J.A., Ellis, I.J., Westbrook, M.T. et Dews, D. (2007). A qualitative study of 369 welfare professionals' perspectives about factors contributing to employee retention and turnover. Children and Youth Services Review, 29, 264-281.

Ferguson, H. (2005). Working with violence, the emotions and the psychosocial dynamics of child protection: Reflections on the Victoria Climbie case. Social Work Education, 24(7), 781-795.

Fernet, C., Austin, S. et Vallerand, R.J. (2012). The effects of work motivation on employee exhaustion and commitment: An extension of the JD-R model. Work \& Stress, 26, 213-219. doi: 10.1080/02678373.2012.713202

Flower, C., McDonald, J. et Sumski, M. (2005). Review of turnover in Milwaukee County private agency child welfare ongoing case management staff. Milwaukee, WI: Governor's Office State of Wisconsin.

Forest, J., Crevier-Braud, L. et Gagné, M. (2009). Mieux comprendre la motivation au travail. Effectif, juin-juillet, 23-27.

Fortin, M.-F. et Gagnon, J. (2016) Fondements et étapes du processus de recherche. Méthodes quantitative et qualitatives ( $3^{\mathrm{e}}$ éd.). Montréal, QC : Chenelière Éducation.

Gagné, M., Chemolli, E., Forest, J. et Koestner, R. (2008). The temporal relations between work motivation and organizational commitment. Psychologica Belgica, 48, 219-241.

Gagné, M. et Deci, E.L. (2005). Selfdetermination theory and work motivation. Journal of Organizational Behavior, 26, 331-362.

Geoffrion, S. (2015). Aggression and accountability: How caregivers and law enforcers cope (Thèse de doctorat inédite). Université de Montréal, Montréal, QC. 
Geoffrion, S., Morselli, C. et Guay, S. (2016). Rethinking compassion fatigue through the lens of professional identity: The case of child-protection workers. Trauma, Violence, \& Abuse, 1(14). doi: $10.1177 / 1524838015584362$

Geoffrion, S. et Ouellet, F. (2013). Quand la réadaptation blesse? Éducateurs victimes de violence. Criminologie, 46(2), 263-289.

Gibbs, A.J. (2001). Maintaining front-line workers in child protection: A case for refocusing supervision. Child Abuse Review, 10, 323-335. doi: 10.1002/ car.707

Gillet, N., Gagné, M., Sauvagère, S. et Fouquereau, E. (2013). The role of supervisor autonomy support, organizational support, and autonomous and controlled motivation in predicting employees' satisfaction and turnover intentions. European Journal of Work and Organizational Psychology, 22, 450-460. doi: 10.1080/1359432X.2012.665228

Gillet, N., Huart, I., Colombat, P. et Fourquereau, E. (2013). Perceived organizational support, motivation, and engagement among police officer. Professional Psychology: Research and Practice, 44(1), 46-55.

Graeff, I.M. et Hill, L.E. (2000). Costing child protective services staff turnover. Child Welfare, 79(5), 517-532.

Güntert, S. (2015). The impact of work design, autonomy support, and strategy on employee outcomes: A differentiated perspective on self-determination at work. Motivation and Emotion, 39, 99103. doi: 10.1007/s11031-014-9412-7

Harnois, G. et Gabriel, P. (2000). Mental health and work: Impact, issues and good practices. Suisse: Organisation mondiale de la santé (OMS).

Horejsl, C., Garthewait, C. et Rolando, J. (1994). A survey of threats and violence directed against child welfare workers in rural state. Child Welfare, 73(2), 173179.
Horwitz, M.J. (2006). Work-related trauma effects in child protection social workers. Journal of Social Service Research, 32(3), 1-18.

Laframboise, A., Laurent, F.-A. et Houlfort, N. (2016). La théorie d'auto-détermination appliquée au travail. Dans Y. Paquet, N. Carbonneau et R.J. Vallerand (dir.), Théorie de l'autodétermination : aspects théoriques et appliqués (p. 229-248). Bruxelles, Belgique : Éditions de Boeck.

Lanctôt, N. et Guay, S. (2014). The aftermath of workplace violence among healthcare workers: A systematic literature review of the consequences. Aggression and Violent Behavior, 19(5), 492-501.

Littlechild, B. (2005a). The stresses arising from violence, threats and aggression against child protection social workers. Journal of Social Work, 5(1), 61-82. doi: $10.1177 / 1468017305051240$

Littlechild, B. (2005b). The nature and effects of violence against child-protection social workers: Providing effective support. British Journal of Social Work, 35(3), 387-401. doi: 10.1093/bjsw/ bch188

Littlechild, B., Hunt, S., Goddard, C., Cooper, J., Raynes, B. et Wild, J. (2016). The effects of violence and aggression from parents on child protection workers' personal, family, and professional lives. Sage Open, 6(1), 1-12. doi: $10.1177 / 2158244015624951$

Macdonald, G. et Sirotich, F. (2005). Violence in the social work workplace the Canadian experience. International Social Work, 48(6), 772-781.

Martinez, C. (2004). Job stress and job satisfaction in child welfare: An analysis of the contributing factors and the impact of supervision. Manitoba: University of Manitoba.

Meadors, P. et Lamson, A. (2008). Compassion fatigue and secondary traumatization: Provider self care on intensive care units for children. Journal of Pediatric Health Care, 22(1), 24-34. 
Miles, M.B., Huberman, A.M. et Saldaña, J. (2014). Qualitative data analysis: A method sourcebook. Thousands Oaks, CA: Sage Publications.

Mor Barak, M.E., Levin, A., Nissly, J.A. et Lane, J.C. (2006). Why do they leave? Modeling child welfare worker's turnover intentions. Children and Youth Services Review, 28, 548-577.

Mor Barak, M.E., Nissly, J.A. et Levin, A. (2001). Antecedents in retention and turnover among child welfare, social work, and other human service employees: What can we learn from past research? A review and metanalysis. The Social Service Review, 75, 625-645.

Nie, Y., Chua, B.L., Yeung, A.S., Ryan, R.M. et Chan, W.Y. (2014). The importance of autonomy support and the mediating role of work motivation for well-being: Testing self-determination theory in a Chinese work organization. International Journal of Psychology, 50, 245-255. doi: 10.1002/ijop.12110

Nix, A.G., Ryan, M.R., Manly, B.J. et Deci, L.E. (1999). Revitalization through selfregulation: The effects of autonomous and controlled motivation on happiness and vitality. Journal of Experimental Social Psychology, 35, 266-284. doi: 10.1007/s11031-011-9250-9

Osofsky, J.D., Putnam, F.W. et Lederman, J.C.S. (2008). How to maintain emotional health when working with trauma. Juvenile and Family Court Journal, 59, 91-102.

Otis, N. et Pelletier, L.G. (2005). A motivational model of daily hassles, physical symptoms, and future work intentions among police officers. Journal of Applied Social Psychology, 35, 21932214.

Paillé, P. et Mucchielli, A. (2016). L'analyse qualitative en sciences humaines et sociales ( $4^{\mathrm{e}}$ éd.). Paris, France : Armand Colin.
Pires, A.P. (1997). Échantillonnage et recherche qualitative: essai théorique et méthodologique. Dans J. Poupart, J.-P. Deslauriers, L.H. Groulx, A. Laperrière, R. Mayer et A.P. Pires (dir.), La recherche qualitative. Enjeux épistémologiques et méthodologiques (p. 113-169). Montréal, QC : Gaëtan Morin Éditeur.

Porter, L.W. et Lawler, E.E. (1968). Managerial attitudes and performance. Homewood, IL: Irwin-Dorsey.

Regehr, C., Hemsworth, D., Leslie, B., Howe, P. et Chau, S. (2004). Predictors of post traumatic distress in child welfare workers: A linear structural equation model. Children and Youth Services Review, 26(4), 331-346.

Rhee Y.S., Ko, Y.B. et Han, I.Y. (2013). Posttraumatic growth and related factors of child protective service workers. Annals of Occupational and Environmental Medicine, 25(6), 1-10.

Richer, S.F., Blanchard, C. et Vallerand, R.J. (2002). A motivational model of work turnover. Journal of Applied Social Psychology, 32, 2089-2113.

Ryan, M.R et Deci, L.E. (2017). Selfdetermination theory basic psychological needs in motivation, development, and wellness. New York, NY: Guilford Press

Tremblay, C. et Joly, J. (2009). Le roulement de personnel chez des intervenants en centre jeunesse : état, causes et effets. Revue de psychoéducation, 38(2), 189213.

Trépanier, S.G., Fernet, C. et Austin, S. (2013). The moderating role of autonomous motivation in the job demands-strain relation: A two sample study. Motivation and Emotion, 37, 93105. doi: $10.1007 / s 11031-012-9290-9$

Turcotte, D. et Pouliot, E. (2006). Les déterminants de la satisfaction au travail chez des intervenants sociaux en protection de l'enfance. Travail social canadien, 8(1), 46-65. 
U.S. Government Accountability Office. Van den Broeck, A., Lens, W., De Witte, H. (2003). Child welfare: HHS could et Van Coillie, H. (2013). Unraveling the play a greater role in helping child importance of the quantity and the quality welfare agencies recruit and retain staff (Publication $\left.\mathrm{n}^{\circ} \mathrm{GAO}-03-357\right)$. Washington, DC: Auteur. of workers' motivation for well-being: A person-centered perspective. Journal of Vocational Behavior, 82, 69-78. doi: 10.1016/j.jvb.2012.11.00510.1016/j.

Vallerand, R. J. et Thill, E.E. (1993). Introduction au concept de motivation. jvb.2012.11.005

Dans R.J. Vallerand et E.E. Thill (dir.), Introduction à la psychologie de la motivation (p. 3-40). Laval, QC : Études Vivantes. 